\title{
THE EXPOSED POINTS OF THE SET OF INVARIANT MEANS
}

\author{
TIANXUAN MIAO
}

\begin{abstract}
Let $G$ be a $\sigma$-compact infinite locally compact group, and let $L I M$ be the set of left invariant means on $L^{\infty}(G)$. We prove in this paper that if $G$ is amenable as a discrete group, then $L I M$ has no exposed points. We also give another proof of the Granirer theorem that the set $\operatorname{LIM}(X, G)$ of $G$-invariant means on $L^{\infty}(X, \beta, p)$ has no exposed points, where $G$ is an amenable countable group acting ergodically as measure-preserving transformations on a nonatomic probability space $(X, \beta, p)$.
\end{abstract}

\section{InTRODUCTION AND Notations}

Let $G$ be a locally compact group with a fixed left Haar measure $\lambda$. If $G$ is compact, we assume $\lambda(G)=1$. Let $L^{p}(G)$ be the associated real Lebesgue spaces $(1 \leq p \leq \infty)$. For $f \in L^{\infty}(G)$ and $x \in G$, the left translation of $f$ by $x$ is defined by ${ }_{x} f(y)=f(x y), y \in G$. A mean on $L^{\infty}(G)$ is a positive functional on $L^{\infty}(G)$ with $m(1)=1$. A left invariant mean is a mean with $m\left({ }_{x} f\right)=m(f)$ for any $x \in G$ and $f \in L^{\infty}(G)$. The set of left invariant mean on $L^{\infty}(G)$ is denoted by $L I M$.

If $L I M \neq \phi$, we say that $G$ is amenable. Let $G_{d}$ be the same algebraic group as $G$ with a discrete topological structure. Then $G$ is amenable if $G_{d}$ is amenable. Properties of amenable groups and left invariant means can be found in Greenleaf [9], Paterson [10] and Pier [11] .

When $G$ is amenable, $L I M$, as a $w^{*}$-compact convex subset of $L^{\infty}(G)^{*}$, is the $w^{*}$-closed convex hull of all its extreme points. It is natural to ask how many exposed points $L I M$ has. Granirer [4] studied intensively the existence of exposed points of $L I M$ for a countable amenable semigroup (also see Chou [1]). In particular, he proved by using very general theorems that $L I M$ has exposed points if and only if $G$ has finite left ideals for a countable amenable semigroup $G$ [4, Corollary 4.1]. Yang [15] proved that if $G$ is a infinite amenable discrete group, then $L I M$ has no exposed points.

In this paper, we prove that $L I M$ has no exposed points for any $\sigma$-compact locally compact group which is amenable as a discrete group. The idea of the proof is to "split" a nonnegative function in $L^{\infty}(G)$ by a category argument,

Received by the editors January 5, 1994; originally communicated to the Proceedings of the AMS by J. Marshall Ash.

1991 Mathematics Subject Classification. Primary 43A07.

Key words and phrases. Locally compact groups, amenable groups, invariant means, the exposed points.

This research is supported by an NSERC grant. 
the technique used by Rosenblatt [12]. We also adapt this technique to prove the Granirer theorem of [5] and [6] in a different way that the set $\operatorname{LIM}(X, G)$ of $G$-invariant means on $L^{\infty}(X, \beta, p)$ has no exposed points, where $G$ is an amenable countable group acting ergodically as measure-preserving transformations on a nonatomic probability space $(X, \beta, p)$. He derives it using very general theorems. See Chou [2] and Rosenblatt [13] for details of the study of the set $\operatorname{LIM}(X, G)$.

The author would like to thank Professor E.E. Granirer for pointing out that Theorem 2 in this paper is a special case of his general theorems in [5] and [6] and for many valuable conversations.

\section{EXPOSED POINTS OF LIM}

In this section we will be concerned with $L I M$ for a locally compact group and will prove our first main result. We need the following, probably known, proposition for which we were unable to find a reference.

Proposition 1. Let $G$ be a nondiscrete locally compact group, and let $K$ be a compact subset of $G$. If $f \in L^{\infty}(G)$ and $\lambda\{t \in G: f(t) \neq 0\}$ is finite, then the function defined by

$$
F\left(x_{1}, x_{2}, \ldots, x_{n}\right)=\lambda\left\{t \in G: \frac{1}{n} \sum_{i=1}^{n}{ }_{x_{i}} f(t)>a\right\}
$$

is lower semicontinuous on $K^{n}$, where $a$ is a constant.

Proof. First let us prove that $\int_{G}\left|{ }_{x} f-f\right| d t \rightarrow 0$ as $x \rightarrow e$. If $f=1_{E}$, then $\left.\int_{G}\right|_{x} f-f \mid d t=\lambda\left(x^{-1} E \Delta E\right) \rightarrow 0$ as $x \rightarrow e$ since the map $x \rightarrow \lambda\left(x^{-1} E \cap E\right)$ is continuous from $K$ to $R$. For any $f$ with $\lambda\{t \in G: f(t) \neq 0\}$ finite and an $\epsilon>0$, choose a simple function $\varphi=\sum_{p=1}^{q} a_{p} 1_{E_{p}}$ such that $\|f-\varphi\|_{1}<\epsilon$. There exists an open neighborhood $U$ of $e$ such that $\sum_{p=1}^{q}\left|a_{p}\right| \int_{G}\left|{ }_{x} 1_{E_{p}}-1_{E_{p}}\right| d t<\epsilon$ for any $x \in U$. Hence, for every $x \in U$,

$$
\int_{G}\left|{ }_{x} f-f\right| d t \leq \int_{G}\left|{ }_{x} f-{ }_{x} \varphi\right| d t+\int_{G}\left|{ }_{x} \varphi-\varphi\right| d t+\int_{G}|\varphi-f| d t \leq 3 \epsilon .
$$

Let $u^{\alpha}=\left(u_{1}^{\alpha}, u_{2}^{\alpha}, \ldots, u_{n}^{\alpha}\right)$ be a net and $u=\left(u_{1}, u_{2}, \ldots, u_{n}\right) \in K^{n}$ with $u^{\alpha} \rightarrow u$ in $K^{n}$. If there is an $\epsilon_{0}>0$ such that $F\left(u^{\alpha}\right)<F(u)-\epsilon_{0}$, then we can find a $\delta>0$ such that $F\left(u^{\alpha}\right)<\lambda\left\{t \in G: \frac{1}{n} \sum_{i=1}^{n} u_{t} f(t)>a+\delta\right\}-\epsilon_{0}$ for every $\alpha$. Thus

$$
\int_{G}\left|\frac{1}{n} \sum_{i=1}^{n} u_{i}^{a} f-\frac{1}{n} \sum_{i=1}^{n}{ }_{u_{t}} f\right| d t \leq \frac{1}{n} \sum_{i=1}^{n} \int_{G}\left|u_{i} f-{ }_{u_{i}} f\right| d t \rightarrow 0
$$

when $u^{\alpha} \rightarrow u$ in $K^{n}$. On the other hand,

$$
\int_{G}\left|\frac{1}{n} \sum_{i=1}^{n} u_{i}^{a} f-\frac{1}{n} \sum_{i=1}^{n} u_{i} f\right| d t \geq \int_{B_{i}}\left|\frac{1}{n} \sum_{i=1}^{n} u_{i}^{a} f-\frac{1}{n} \sum_{i=1}^{n} u_{i} f\right| d t \geq \delta \lambda\left(B_{\alpha}\right) \geq \delta \epsilon_{0},
$$

where

$$
B_{\alpha}=\left\{t \in G: \frac{1}{n} \sum_{i=1}^{n} u_{i} f(t)>a+\delta\right\} \sim\left\{t \in G: \frac{1}{n} \sum_{i=1}^{n} u_{i}^{n} f(t)>a\right\}
$$


with $\lambda\left(B_{\alpha}\right) \geq \lambda\left\{t \in G: \frac{1}{n} \sum_{i=1}^{n} u_{i} f(t)>a+\delta\right\}-F\left(u^{\alpha}\right)>\epsilon_{0}$. This is a contradiction. Therefore the function $F$ from $K^{n}$ to $R$ is lower semicontinuous.

To prove our result, we will need the following two lemmas.

Lemma 2. Let $G$ be a locally compact group and let $f \in L^{\infty}(G)$ be a function with $0 \leq f \leq 1$ and $\lambda\{x \in G: f(x) \neq 0\}<\infty$. If $f_{k}$ is a sequence of functions in $L^{\infty}(G)$ with $0 \leq f_{k} \leq f \quad(k=0,1,2, \ldots), \quad f_{k} \rightarrow f_{0}$ in $\|\cdot\|_{1}$-norm, then $\inf _{\left(x_{1}, x_{2}, \ldots, x_{n}\right) \in K^{n}} F_{f_{k}}\left(x_{1}, x_{2}, \ldots, x_{n}\right)=0$ for any $k \geq 1$ implies $\inf _{\left(x_{1}, x_{2}, \ldots, x_{n}\right) \in K^{n}} F_{f_{0}}\left(x_{1}, x_{2}, \ldots, x_{n}\right)=0$, where $K$ is a compact subset of $G$ and $F_{h}\left(x_{1}, x_{2}, \ldots, x_{n}\right)=\lambda\left\{t \in G: \frac{1}{n} \sum_{i=1}^{n} x_{i} h(t)>a\right\}$ for any $h \in L^{\infty}(G)$. Proof. Let $\inf _{\left(x_{1}, x_{2}, \ldots, x_{n}\right) \in K^{n}} F_{f_{0}}\left(x_{1}, x_{2}, \ldots, x_{n}\right)=\epsilon_{0}>0$. Then for any $x=$ $\left(x_{1}, x_{2}, \ldots, x_{n}\right) \in K^{n}$, there is an $i_{x} \in N$ such that $\lambda\left\{t \in G: \frac{1}{n} \sum_{i=1}^{n} x_{i} f_{0}(t)\right.$ $\left.>a+\frac{1}{i_{x}}\right\}>\frac{\epsilon_{0}}{2}$. Since the map $\left(y_{1}, y_{2}, \ldots, y_{n}\right) \rightarrow \lambda\left\{t \in G: \frac{1}{n} \sum_{i=1}^{n} y_{i} f_{0}(t)>\right.$ $\left.a+\frac{1}{i_{x}}\right\}$ from $K^{n}$ to $R$ is lower semicontinuous by Proposition 1, there exists an open neighborhood $U_{x}$ of $x$ in $K^{n}$ such that $\lambda\left\{t \in G: \frac{1}{n} \sum_{i=1}^{n} y_{i} f_{0}(t)>\right.$ $\left.a+\frac{1}{i_{x}}\right\}>\frac{\epsilon_{0}}{2}$ for any $y=\left(y_{1}, y_{2}, \ldots, y_{n}\right) \in U_{x}$. Let $U_{x^{(1)}}, U_{x^{(2)}}, \ldots, U_{x^{(p)}}$ be a cover of $K^{n}$. Then for any $y \in K^{n}, \lambda\left\{t \in G: \frac{1}{n} \sum_{i=1}^{n} y_{i} f_{0}(t)>a+\frac{1}{i_{0}}\right\}>\frac{\epsilon_{0}}{2}$, where $i_{0}=\max \left\{i_{x^{(1)}}, i_{x^{(2)}}, \ldots, i_{x^{(p)}}\right\}$.

By the hypothesis and the fact that $K^{n}$ is compact, for each $k \in N$, we can choose an $x^{k}=\left(x_{1}^{k}, x_{2}^{k}, \ldots, x_{n}^{k}\right) \in K^{n}$ such that $\lambda\left\{t \in G: \frac{1}{n} \sum_{i=1}^{n} x_{i}^{k} f_{k}(t)>\right.$ $a\}=0$. Then

$$
\int_{G}\left|\frac{1}{n} \sum_{i=1}^{n} x_{i}^{k} f_{0}(t)-\frac{1}{n} \sum_{i=1}^{n} x_{i}^{k} f_{k}(t)\right| d t \leq\left\|f_{0}-f_{k}\right\|_{1} .
$$

On the other hand,

$$
\begin{aligned}
\int_{G}\left|\frac{1}{n} \sum_{i=1}^{n} x_{i}^{k} f_{0}(t)-\frac{1}{n} \sum_{i=1}^{n} x_{i}^{k} f_{k}(t)\right| d t \\
\quad=\int_{\left\{t \in G: \frac{1}{n} \sum_{i=1}^{n} x_{i}^{k} f_{k}(t) \leq a\right\}}\left|\frac{1}{n} \sum_{i=1}^{n} x_{i}^{k} f_{0}(t)-\frac{1}{n} \sum_{i=1}^{n} x_{i}^{k} f_{k}(t)\right| d t \\
\quad \geq \frac{1}{i_{0}} \lambda\left\{t \in G: \frac{1}{n} \sum_{i=1}^{n} x_{i}^{k} f_{0}(t)>a+\frac{1}{i_{0}}\right\} \\
\quad \geq \frac{1}{i_{0}} \frac{\epsilon_{0}}{2} .
\end{aligned}
$$

This contradicts to that $\left\|f_{k}-f_{0}\right\|_{1} \rightarrow 0$.

The following lemma is a consequence of Lemma $6 \mathrm{~A}$ and Lemma $6 \mathrm{C}$ of Talagrand [14].

Lemma 3. Let $G$ be a $\sigma$-compact nondiscrete locally compact group. If $G$ is amenable as a discrete group and $f \in L^{\infty}(G)$, then for any $\epsilon>0$ there is an open subset $\Omega$ of $G$ and an $m_{0} \in L I M$ such that $\lambda(\Omega)<\epsilon, m_{0}\left(1_{\Omega}\right)=1$, and $m_{0}(f)=\operatorname{Sup}\{m(f): m \in L I M\}$.

Proof. It follows from step 1 of the proof of Theorem 6D in [14] that for any positive integer $n$, there exists an open set $\Omega_{n}$ and an $m_{n} \in L I M$ such that 
$\lambda\left(\Omega_{n}\right)<\frac{\epsilon}{2^{n}}, m_{n}\left(1_{\Omega_{n}}\right)=1$, and $m_{n}(f) \geq \operatorname{Sup}\{m(f): m \in L I M\}-\frac{1}{n}$ (in fact, the condition of that $\nu$ is a topologically left invariant is not used and $\lambda(\Omega)$ can be made as small as we want in the proof of step 1).

Let $m_{0}$ be a $w^{*}$-limit point of $\left\{m_{n}\right\}$ and $\Omega=\bigcup_{n=1}^{\infty} \Omega_{n}$. Then $\lambda(\Omega)<\epsilon$, $m_{0} \in L I M$ with $m_{0}\left(1_{\Omega}\right)=1$, and $m_{0}(f)=\operatorname{Sup}\{m(f): m \in L I M\}$.

Now we are ready to prove our first main result concerning the exposed points of $L I M$ for a locally compact group.

Theorem 1. Let $G$ be a $\sigma$-compact infinite locally compact group. If $G$ is amenable as a discrete group, then LIM has no exposed points.

Proof. When $G$ is discrete, it is proved by Yang [15] that $L I M$ has no exposed points. Assume that $G$ is nondiscrete. Since $G$ is $\sigma$-compact, there is a sequence of subsets $\left\{K_{n}: n \in N\right\}$ such that $G=\bigcup_{n=1}^{\infty} K_{n}$, where $K_{n}$ is compact and $K_{n} \subseteq K_{n+1} \quad(n=1,2, \ldots)$. Assume that $m_{0} \in L I M$ is an exposed point of $L I M$. Then there is an $f_{0} \in L^{\infty}(G)$ such that

$$
m_{0}\left(f_{0}\right)>m\left(f_{0}\right) \text { for any } m \in L I M \text { and } m \neq m_{0} \text {. }
$$

We are going to show that we can choose $f_{0}$ as above such that $0 \leq f_{0} \leq 1$ and $f_{0} \in L^{1}(G)$. Let $f_{1}=\frac{f_{0}+\left\|f_{0}\right\|_{\infty}}{\left\|f_{0}+\right\| f_{0}\left\|_{\infty}\right\|_{\infty}}$. Then $f_{1}$ also satisfies $(*)$ since $f_{1} \geq 0$ and $m(1)=1$ for all $m \in L I M$. Thus, $m_{0}\left(f_{1}\right)>0$ by the fact that $L I M \neq\left\{m_{0}\right\}$ (see [7]). By Lemma 3, there exists an open subset $\Omega$ of $G$ and an $m_{1} \in L I M$ such that $\lambda(\Omega)<1, m_{1}\left(1_{\Omega}\right)=1$, and $m_{1}\left(f_{1}\right)=\operatorname{Sup}\left\{m\left(f_{1}\right): m \in L I M\right\}$. Hence $m_{1}\left(f_{1}\right)=m_{0}\left(f_{1}\right)$ and $m_{1}=m_{0}$ by $(*)$. Let $g=f_{1} 1_{\Omega}$. Then $g$ satisfies $(*)$. In fact, for any $m \in L I M \sim\left\{m_{0}\right\}, m(g)=m\left(f_{1} 1_{\Omega}\right) \leq m\left(f_{1}\right)<$ $m_{0}\left(f_{1}\right)=m_{0}(g)$ since $m_{0}\left(f_{1} 1_{G \sim \Omega}\right)=m_{1}\left(f_{1} 1_{G \sim \Omega}\right)=0$. Note that $g \geq 0$ and $g \in L^{\infty}(G) \cap \mathrm{L}^{1}(G)$. Let $X=\left\{f \in L^{\infty}(G): 0 \leq f \leq g\right\}$ and $a=m_{0}(g)$. Then $\left(X,\|\cdot\|_{1}\right)$ is a complete metric space and $a>0$.

Let $n \in N$ and $n>0$ be fixed. For any $p, q \in N$, put

$$
\begin{aligned}
& X_{p, q}=\left\{f \in X: \exists x_{1}, x_{2}, \ldots, x_{p} \in K_{q}\right. \\
& \left.\quad \text { with } \lambda\left\{t \in G: \frac{1}{p} \sum_{i=1}^{p} x_{i} f(t)>a-\frac{1}{n}\right\}=0\right\} .
\end{aligned}
$$

At first, each $X_{p, q}$ is closed. In fact, let $f_{k} \in X_{p, q}$ and $f_{k} \rightarrow f$ in $\left(X,\|\cdot\|_{1}\right)$. By Lemma 2,

$$
\inf _{\left(x_{1}, x_{2}, \ldots, x_{p}\right) \in K_{q}^{p}} \lambda\left\{t \in G: \frac{1}{p} \sum_{i=1}^{p} x_{i} f(t)>a-\frac{1}{n}\right\}=0 .
$$

By Lemma 1, the map $\left(x_{1}, x_{2}, \ldots, x_{p}\right) \rightarrow \lambda\left\{t \in G: \frac{1}{p} \sum_{i=1}^{p} x_{i} f(t)>a-\frac{1}{n}\right\}$ from $K_{q}^{p}$ to $R$ is lower semicontinuous. Since $K_{q}^{p}$ is compact, there exists $\left(x_{1}, x_{2}, \ldots, x_{p}\right) \in K_{q}^{p}$ such that $\lambda\left\{t \in G: \frac{1}{p} \sum_{i=1}^{p} x_{i} f(t)>a-\frac{1}{n}\right\}=0$. Therefore $f \in X_{p, q}$.

Also, $X_{p, q}$ is nowhere dense. In fact, for any $f \in X$ and any $\epsilon>0$, by Lemma 3 there is an open subset $\Omega_{1}$ of $G$ and an $m_{1} \in L I M$ such that $\lambda\left(\Omega_{1}\right)<\epsilon, m_{1}\left(1_{\Omega_{1}}\right)=1$, and $m_{1}(g)=\operatorname{Sup}\{m(g): m \in L I M\}$. Since 
$g$ satisfies $(*), m_{1}=m_{0}$. Let $f^{*}=g 1_{\Omega_{1}}+f 1_{G \sim \Omega_{1}}$. Then $f^{*} \in X$ and $\left\|f^{*}-f\right\|_{1}=\left\|g 1_{\Omega_{1}}-f 1_{\Omega_{1}}\right\|_{1}<2 \epsilon$. Since $m_{0}\left(f^{*}\right)=m_{0}\left(g 1_{\Omega_{1}}\right)=m_{0}(g)=a>$ $a-\frac{1}{n}, \lambda\left\{t \in G: \frac{1}{p} \sum_{i=1}^{p} x_{i} f^{*}(t)>a-\frac{1}{n}\right\} \neq 0$ for any $\left(x_{1}, x_{2}, \ldots, x_{p}\right) \in K_{q}^{p}$. Hence $f^{*} \notin X_{p, q}$.

For any $p, q \in N$, let $X_{p, q}^{c}=\left\{f \in X: g-f \in X_{p, q}\right\}$. Then $X_{p, q}$ and $X_{p, q}^{c}$ are isometric in $\left(X,\|\cdot\|_{1}\right)$. So $X_{p, q}^{c}$ is also nowhere dense in $\left(X,\|\cdot\|_{1}\right)$. Hence there exists an $f \in X \sim \bigcup_{p, q}\left(X_{p, q} \cup X_{p, q}^{c}\right)$ by the completeness of $X$.

For any $x_{1}, x_{2}, \ldots, x_{p} \in G$, there is $q \in N$ such that $x_{1}, x_{2}, \ldots, x_{p} \in K_{q}$. Thus, $\lambda\left\{t \in G: \frac{1}{p} \sum_{i=1}^{p} x_{i} f(t)>a-\frac{1}{n}\right\} \neq 0$ since $f \notin X_{p, q}$. There exists $m_{n} \in L I M$ such that $m_{n}(f)>a-\frac{1}{n}$ by Proposition 3 of [7]. Similarly, since for any $x_{1}, x_{2}, \ldots, x_{p} \in G, \lambda\left\{t \in G: \frac{1}{p} \sum_{i=1}^{p} x_{i}(g-f)(t)>a-\frac{1}{n}\right\} \neq 0$, there exists $M_{n} \in L I M$ such that $M_{n}(g-f)>a-\frac{1}{n}$. Let $m$ and $M$ be $w^{*}$ limit points of $m_{n}$ and $M_{n}$, respectively. Then $m, M \in L I M$ and $m(f) \geq a$ and $M(g-f) \geq a$. Since $0 \leq f \leq g$ and $0 \leq g-f \leq g, m(g) \geq a$ and $M(g) \geq a$. Hence $m=M=m_{0}$ by $(*)$ and since $a=m_{0}(g)=\operatorname{Sup}\{m(g): m \in L I M\}$. Therefore $M(g-f)=0$. This contradicts $a>0$.

\section{EXPOSED POINTS OF $\operatorname{LIM}(X, G)$}

In this section we are going to prove an analogue of Theorem 1 for groups acting ergodically as measure-preserving transformations on a nonatomic probability space $(X, \beta, p)$.

Let $(X, \beta, p)$ be a nonatomic probability space, $G$ a group, and $(s, x) \rightarrow$ $s x$ a measure-preserving ergodic action of $G$ on $(X, \beta, p)$. Then $G$ also acts on $L^{\infty}(X, \beta, p):(s f)(x)=f(s x), \quad f \in L^{\infty}(X, \beta, p), \quad s \in G$, and $x \in X$. A positive linear functional of norm 1 on $L^{\infty}(X, \beta, p)$ is said to be $G$-invariant mean if $m(s f)=m(f)$ for $s \in G$ and $f \in L^{\infty}(X, \beta, p)$. The set of $G$-invariant means is denoted by $\operatorname{LIM}(X, G)$.

It is natural to ask how big the set $\operatorname{LIM}(X, G)$ is. When $G$ is a countable amenable semigroup, del Junco and Rosenblatt [3] proved $\operatorname{LIM}(X, S)$ contains more than one element. Chou [2] showed that the cardinality of $\operatorname{LIM}(X, G)$ is at least $2^{c}$ for any countable amenable group, where $c$ is the cardinality of the continuum. Our Theorem 2 shows that $\operatorname{LIM}(X, G)$ does not have exposed points in the case that $G$ is an amenable countable group acting ergodically as measure-preserving transformations on a nonatomic probability space. This theorem was proved by Granirer in Theorem 3 in [5] and Theorem 2.6 in [6] without the assumptions of the ergodical acting and the measure-preserving transformations. Here we will give a different and direct proof.

Lemma 4. Let $G$ be a group acting ergodically as measure-preserving transformations on a nonatomic probability space $(X, \beta, p)$. If $m \in L I M(X, G)$ and $f \in L^{\infty}(X)$ with $0 \leq f \leq 1$, then for any $x_{1}, x_{2}, \ldots, x_{n} \in G, \epsilon>0$, and $\delta>0$ there exists a subset $V$ of $X$ such that $p(V)<\epsilon$ and

$$
p\left\{t \in X: \frac{1}{n} \sum_{i=1}^{n}{ }_{x_{i}}\left(f 1_{V}\right)(t)>m(f)-\delta\right\} \neq 0 .
$$

Proof. Let $a=m(f)$. Since $m\left(\frac{1}{n} \sum_{i=1}^{n} x_{i} f\right)=a, p\left\{t \in X: \frac{1}{n} \sum_{i=1}^{n} x_{i} f(t)>\right.$ $a-\delta\}>0$. Hence there is a subset $J \subseteq\{1,2, \ldots, n\}$ and $a_{i}$ for each $i \in J$ 
such that $\frac{1}{n} \sum_{i \in J} a_{i}>a-\delta$ and $p\left(\bigcap_{i \in J}\left\{t \in X:{ }_{x_{i}} f(t)>a_{i}\right\}\right)>0$. Let $E_{a_{i}}=$ $\left\{t \in X: f(t)>a_{i}\right\}$. Then $\left\{t \in X:{ }_{x_{i}} f(t)>a_{i}\right\}=\left\{t \in X: x_{i} t \in E_{a_{i}}\right\}$, which is denoted by $x_{i}^{-1} E_{a_{i}}$. Hence $p\left(\bigcap_{i \in J} x_{i}^{-1} E_{a_{i}}\right)>0$. Since $X$ is nonatomic, there exists $A \subseteq \bigcap_{i \in J} x_{i}^{-1} E_{a_{i}}$ such that $0<p(A)<\frac{1}{n} \epsilon$. Let $V=\bigcup_{i \in J} x_{i} A$. Then $0<p(V)<\epsilon$. If $t \in A$, then $x_{i} t \in V \cap E_{a_{i}}$ for each $i \in J$. Hence $x_{i}\left(f 1_{V}\right)(t)=1_{V}\left(x_{i} t\right) f\left(x_{i} t\right)>a_{i}$, i.e. $A \subseteq \bigcap_{i \in J}\left\{t \in X: x_{i}\left(f 1_{V}\right)(t)>a_{i}\right\}$ and

$$
\begin{aligned}
0 & <p(A) \\
& \leq p\left\{t \in X: \frac{1}{n} \sum_{i \in J} x_{i}\left(f 1_{V}\right)(t)>a-\delta\right\} \\
& \leq p\left\{t \in X: \frac{1}{n} \sum_{i=1}^{n} x_{i}\left(f 1_{V}\right)(t)>a-\delta\right\} .
\end{aligned}
$$

The following lemma is due to Granirer. See [7, Proposition 3] and [8, Proposition 5] for its proof.

Lemma 5. Let $G$ be a group acting ergodically as measure-preserving transformations on a nonatomic probability space $(X, \beta, p)$. If $m \in \operatorname{LIM}(X, G)$ and $f \in L^{\infty}(X)$, then

$$
\sup \{m(f): m \in \operatorname{LIM}(X, G)\}=\inf _{x_{1}, x_{2}, \ldots, x_{n} \in G} \text { ess } \sup _{t}\left[\frac{1}{n} \sum_{i=1}^{n} x_{i} f(t)\right] .
$$

Theorem 2 (Granirer). If $G$ is a amenable countable group acting ergodically as measure-preserving transformations on a nonatomic probability space $(X, \beta, p)$, then the set $\operatorname{LIM}(X, G)$ of $G$-invariant means on $L^{\infty}(X, \beta, p)$ has no exposed points.

Proof. Let $G=\bigcup_{n=1}^{\infty} K_{n}$, where each $K_{n}$ is a finite subset of $G$ and $K_{n} \subseteq K_{n+1}$ $(n=1,2, \ldots)$. Let $m_{0} \in \operatorname{LIM}(X, G)$ be an exposed point of $\operatorname{LIM}(X, G)$. Then there is an $f_{0} \in L^{\infty}(X)$ such that

$$
m_{0}\left(f_{0}\right)>m\left(f_{0}\right) \text { for any } m \in \operatorname{LIM}(X, G) \text { and } m \neq m_{0} \text {. }
$$

Let $g=\frac{f_{0}+\left\|f_{0}\right\|_{\infty}}{\left\|f_{0}+\right\| f_{0}\left\|_{\infty}\right\|_{\infty}}$. Then $g$ also satisfies $(*)$ since $m(1)=1$ for any $m \in L I M(X, G)$. Note that $g \in L^{\infty}(X) \cap L^{1}(X)$ and $g \geq 0$. Thus $m(g) \geq 0$. for any $m \in \operatorname{LIM}(X, G)$. By $(*)$ and the fact that $M(X, G)$ contains more than one element (see del Junco and Rosenblatt [3]), $m_{0}(g)>0$. Let $a=m_{0}(g)$ and $Y=\left\{f \in L^{\infty}(X): 0 \leq f \leq g\right\}$. Then $\left(Y,\|\cdot\|_{1}\right)$ is a complete metric space and $a>0$. For any $n \in N$ and $\delta>0$, set $X_{n}=\left\{f \in Y: \exists x_{1}, x_{2}, \ldots, x_{k} \in K_{n}\right.$ with $\left.p\left\{t \in X: \frac{1}{k} \sum_{i=1}^{k} x_{i} f(t)>a-\delta\right\}=0\right\}$. At first, each $Y_{n}$ is closed. In fact, let $f_{k} \in Y_{n}$ and $f_{k} \rightarrow f$ in $\left(Y,\|\cdot\|_{1}\right)$. We can assume that $f_{k} \rightarrow f$ a.e. [p]. So for any $x_{1}, x_{2}, \ldots, x_{r} \in K_{n}$

$$
\frac{1}{r} \sum_{i=1}^{r} x_{i} f_{k}(t) \rightarrow \frac{1}{r} \sum_{i=1}^{r} x_{i} f(t) \quad \text { a.e. [p] as } k \rightarrow \infty .
$$

Also, for each $k$, there are $x_{1}, x_{2}, \ldots, x_{r} \in K_{n}$ such that

$$
p\left\{t \in X: \frac{1}{r} \sum_{i=1}^{r}{ }_{x_{i}} f_{k}(t)>a-\delta\right\}=0 .
$$


Since $K_{n}$ is finite, there are $x_{1}, x_{2}, \ldots, x_{r} \in K_{n}$ such that

$$
p\left\{t \in X: \frac{1}{r} \sum_{i=1}^{r} x_{i} f(t)>a-\delta\right\}=0 .
$$

Thus, $f \in Y_{n}$. Therefore $Y_{n}$ is closed.

Also, for any $f \in Y$ and any $\epsilon>0$, for any $x_{1}, x_{2}, \ldots, x_{r} \in K_{n}$, by Lemma 4, there is a subset $V$ of $X$ such that $p(V)<\epsilon$ and

$$
p\left\{t \in X: \frac{1}{r} \sum_{i=1}^{r} x_{i}\left(g 1_{V}\right)(t)>a-\delta\right\}>0 .
$$

Let $f^{*}=g 1_{V}+f 1_{X \sim V}$. Then $f^{*} \in Y$ and $\left\|f^{*}-f\right\|_{1}=\left\|g 1_{V}-f 1_{V}\right\|_{1}<2 \epsilon$. Since $f 1_{X \sim V} \geq 0$,

$$
0<p\left\{t \in X: \frac{1}{r} \sum_{i=1}^{r} x_{i}\left(g 1_{V}\right)(t)>a-\delta\right\} \leq p\left\{t \in X: \frac{1}{r} \sum_{i=1}^{r} x_{i} f^{*}(t)>a-\delta\right\} .
$$

Hence $f^{*} \notin Y_{n}$ and $Y_{n}$ is nowhere dense.

For any $n \in N$, let $Y_{n}^{c}=\left\{f \in Y: g-f \in Y_{n}\right\}$. Then $Y_{n}$ and $Y_{n}^{c}$ are isometric in $\left(Y,\|\cdot\|_{1}\right)$. So $Y_{n}^{c}$ is also nowhere dense in $\left(Y,\|\cdot\|_{1}\right)$. Hence there exists an $f \in Y \sim \bigcup_{n}\left(Y_{n} \cup Y_{n}^{c}\right)$ by the completeness of $Y$.

For any $x_{1}, x_{2}, \ldots, x_{n} \in G$, since $p\left\{t \in X: \frac{1}{n} \sum_{i=1}^{n} x_{i} f(t)>a-\delta\right\}>0$, by Lemma 5 there exist $m_{\delta} \in \operatorname{LIM}(X, G)$, such that $m_{\delta}(f)>a-\delta$. Let $m$ be the $w^{*}$ limit point of $\left\{m_{\delta}\right\}$. Then $m \in \operatorname{LIM}(X, G)$ and $m(f) \geq a$. Similarly, since for any $x_{1}, x_{2}, \ldots, x_{n} \in G, p\left\{t \in X: \frac{1}{n} \sum_{i=1}^{n} x_{i}(g-f)(t)>a-\delta\right\}>0$, there exists $M \in \operatorname{LIM}(X, G)$ such that $M(g-f) \geq a$. Since $0 \leq f \leq g$, $m(g) \geq a$ and $M(g) \geq a$. By $(*), m=M=m_{0}$. So $M(g-f)=0$. This contradicts $a>0$.

\section{REFERENCES}

1. C. Chou, On a geometric property of the set of invariant means on a group, Proc. Amer. Math. Soc. 30 (1971), 296-302.

2. (1987), 647-650.

3. A. del Junco and J. Rosenblatt, Counterexamples in ergodic theory and number theory, Math. Ann. 245 (1979), 185-197.

4. E.E. Granirer, Exposed points of convex sets and weak sequential convergence, Mem. Amer. Math. Soc., vol. 123, Amer. Math. Soc., Providence, RI, 1972.

5. __ Geometric and topological properties of certain $w^{*}$ compact convex sets which arise from the study of invariant means, Canad. J. Math. 37 (1985), 107-121.

6. Geometric and topological properties of certain $w^{*}$ compact convex subsets of double duals of Banach spaces, which arise from the study of invariant means, Illinois J. Math. 30 (1986), 148-174.

7. __ Criteria for compactness and for discreteness of locally compact amenable groups, Proc. Amer. Math. Soc. 40 (1973), 615-624.

8. $\ldots$, On finite equivalent invariant measures for semigroups of transformations, Duke Math. J. 38 (1971), 395-408.

9. F.P. Greenleaf, Invariant means on topological groups, Van Nostrand, New York, 1969.

10. A.L.T. Paterson, Amenability, Amer. Math. Soc., Providence, RI, 1988. 
11. J.P. Pier, Amenable locally compact groups, Wiley, New York, 1984.

12. J.M. Rosenblatt, Invariant means and invariant ideals in $L_{\infty}(G)$ for a locally compact group $G$, J. Funct. Anal. 21 (1976), 31-51.

13. Uniqueness of invariant means for measure preserving transformations, Trans. Amer. Math. Soc. 265 (1981), 623-636.

14. M. Talagrand, Géométrie des simplexes de moyennes invariantes, J. Funct. Anal. 34 (1979), 304-337.

15. Z. Yang, Exposed points of left invariant means, Pacific J. Math. 125 (1986), 487-494.

Department of Mathematical Sciences, Lakehead University, Thunder Bay, Ontario P7E 5E1 CANADA

E-mail address: tmiao@thunder. lakeheadu.ca 